\title{
Association of HLA-DRB1 and HLA-DQB1 alleles with red blood cell auto-immunization in the Chinese population
}

\author{
Yushiang Lin ${ }^{1 \Delta}$, Zhiyuan $\mathrm{Xu}^{2 \Delta}$, Xiaofei $\mathrm{Li}^{2}$, Xiaoyan Shan², Daowang Fan², Yan Qiu ${ }^{2}$, Haochun Chang ${ }^{2}$, \\ Tianhong Miao ${ }^{2 *}$ \\ ${ }^{1}$ Department of Clinical Medicine, School of Basic Medical Sciences, Peking University Health Science Center, Beijing 100191, China; \\ ${ }^{2}$ Blood Group Lab, Beijing Red Cross Blood Center, Beijing 100088, China
}

\begin{abstract}
Red blood cell (RBC) auto-immunization poses a clinical dilemma, because the formation of RBC autoantibodies in certain patients is a significant causative factor for autoimmune hemolytic anemia. To clarify whether specific HLA-DRB1 and HLA-DQB1 alleles are associated with the response to RBC auto-immunization, we conducted a case-controlled retrospective study. The case group included 117 patients with autoantibodies potentially harmful to RBC after transfusion at the Beijing Red Cross Blood Center, and the controls were 1.6 million healthy subjects whose data are stored in the China Marrow Donor Program database. In the case group, the frequencies of HLA-DRBI and HLA-DQB1 allele were determined by PCR-rSSO Luminex high-resolution technology. HLA-DRB1*13 allele expression was not detected in the 75 autoantibody positive patients with antiC3d production (adjusted $P=0.0383$ ), whereas the presence of HLA-DRB1*14 allele showed a positive correlation with the autoantibody positive group with anti-C3d production (odds ratio $=2.22,95 \%$ confidence interval $=1.29-3.84$, adjusted $P=0.0383$ ). The HLA-DQB1 $* 02$ allele was more frequent in patients with both RBC autoantibodies and alloantibodies. There was no significant association between a positive antiglobulin test result and the presence of HLA-DRB1 and HLA-DQB 1 alleles. In summary, we found that HLA-DRB1*13 may serve as a protective factor, whereas HLA-DRB $1 * 14$ appears to be a risk factor for the production of autoantibodies positive with anti-C3d in RBC auto-immunization. To our knowledge, this is the first study to propose HLA-DRB1*13 as a protective factor in auto-immunization.
\end{abstract}

Keywords: HLA-DRB1, HLA-DQB1, red blood cell auto-immunization, autoantibody

\section{INTRODUCTION}

The development of autoantibodies may destroy red blood cells (RBCS), leading to autoimmune hemolytic anemia (AIHA) along with clinical evidence of hemolysis and anemia ${ }^{[1]}$. Patients with autoimmune diseases (such as systemic lupus erythematosus), infected by

\footnotetext{
*Correspondence to: Tianhong Miao, Blood Group Lab, Beijing Red Cross Blood Center, Beijing 100088, China. TEL: +86-10-82807272; E-mail: tianhongm@163.com.

${ }^{\Delta}$ These authors contributed equally to this work.
}

Mycoplasma pneumoniae, or those receiving repeated transfusions due to leukemia hemoglobinopathies (including thalassemia and sickle cell anemia), are at particularly high risk for the development of RBC autoantibodies ${ }^{[1,2]}$. Although RBC autoantibodies are not always associated with AIHA, or present in all hemolytic clinical conditions, RBC autoantibodies causes huge problems in blood transfusion practice, resulting in incompatibility for major and minor cross-match results ${ }^{[3]}$. AIHA is estimated to occur in approximately 1 in 80,000 patients receiving transfusions annually, and a large study conducted in China demonstrated a frequency of $29.5 \%$ autoantibodies among all unex- 
pected antibodies ${ }^{[3,4]}$.

RBC autoantibodies are usually detected by either an antibody screening test or direct anti-globulin test (DAT), followed by further examination to distinguish between warm type $\left(\mathrm{DAT}^{+}\right.$for IgG only or for IgG and $\mathrm{C} 3 \mathrm{~d}$, and a positive reaction of the plasma with the screen and panel cells at the warm phase), cold agglutinin type (DAT ${ }^{+}$for C3d only, and a positive reaction of the plasma with the screen and panel cells at room temperature only), and mixed type (DAT ${ }^{+}$for both IgG and C3d, with co-existing cold agglutinins and warm antibodies) ${ }^{[2]}$.

Data from clinical transfusion practice have revealed strong associations between certain HLADRBl genotypes and the presence of RBC alloantibodies, and patients showing responses to multiple antibodies (i.e., multi-responders), with detection of specific alloantibodies following immunization by only a single transfusion, demonstrated differential HLA-DRB 1 alleles ${ }^{[5]}$. Only one study has thus far demonstrated relationships between the HLA-DQB 1 alleles and RBC allo-immunization ${ }^{[6]}$. However, there is less information available on the potential role of HLA class II status in the development of RBC autoantibodies. Therefore, the aim of our study was to investigate the associations between HLA-DRB1/ HLA-DQB1 alleles and the development of RBC autoantibodies of different DAT types $\left(\mathrm{C}_{3} \mathrm{~d}^{+}, \mathrm{IgG}^{+}\right.$, and $\mathrm{C} \mathrm{d}^{+} \mathrm{IgG}^{+}$) using a large dataset from the Chinese population. These results are expected to clarify the role of HLA status in RBC immunization and to identify candidate biomarkers for assuring safe transfusion practice.

\section{MATERIALS AND METHODS}

\section{Study population}

Data were collected retrospectively from patients who received RBC transfusion and showed positive antibody screen test results from the North Blood Grouping Reference Lab (BGRL) and the Beijing Red Cross Blood Center (BRCBC) of China from December 2014 to December 2015. The case group included 117 patients identified with RBC autoantibodies based on the presence of plasma clumps reacting with all screen cells and panel cells and a positive DAT test result, including 9 patients with RBC autoantibodies plus allo-anti-Rh (5 patients with anti-E, 3 patients with anti-c, and one patient with anti-c, E). Data of the control group were collected from the China Bone Marrow Donor Registry Program database, including information on 1.6 million healthy Chinese subjects ${ }^{[7]}$.

\section{Antibody screen test, DAT, and antibody identification}

The screen and identification cells used at the BGRL of the BRCBC cover all the major antigen systems, including Rh, MNS, Duffy, Kidd, Kell, Lewis, Pl, Xg, and Lutheran, as well as lowfrequency antigens such as Dia and Mur (Bio-Rad $\mathrm{GmbH}$, Germany and Sanquin GmbH, Switzerland), which have a relatively high prevalence in Asia. The main antibody detection methods include conventional tube techniques conducted at room temperature and gel column tests(Bio-Rad GmbH, Germany). All of the data in the present study were based on a gel column test to screen for antibodies. Only a few individual samples that showed negative results in the gel column test were identified as positive in the room temperature tube test. The other procedures, such as phenotyping, sample reception, report documentation, and report writing were all based on regulations and the standard procedures specified by the BGRL of the BRCBC.

\section{DNA extraction and genotyping of the HLA- DRB1 and HLA-DQB1 alleles}

DNA was isolated from ethylene diaminetetraacetic acid-anti-coagulated blood using a commercial kit (Prepito DNA Blood 250 Kit, Chemagen, PerkinElmer, Germany) based on magnetic separation on an automated system (Chemagic Prepito, Chemagen, PerkinElmer, Germany). All DNA samples were stored at $-80^{\circ} \mathrm{C}$ until molecular analysis. HLA-DRB1 and HLA-DQB1 genotyping was performed with high-resolution DRB1-SSO, DQB1-SSO commercial kits (LABType rSSO, One Lambda, Canoga Park, CA).

\section{Grouping and data analyses}

The patients were grouped as DAT test result, total patients $(n=117), \operatorname{IgG}^{+}$(i.e., autoantibodies positive with anti-IgG without reference to anti-C3d, $n=113), \mathrm{C}^{+} \mathrm{d}^{+}($i.e., autoantibodies positive with antiC3d without reference to anti-IgG, $n=77), \mathrm{IgG}^{+} / \mathrm{C} 3 \mathrm{~d}^{-}$ (autoantibodies positive with anti-IgG and negative with anti-C $3 \mathrm{~d}, n=40$ ), $\mathrm{IgG}^{-} / \mathrm{C}^{2} \mathrm{~d}^{+}$(autoantibodies negative with anti- $\operatorname{IgG}$ and positive with anti-C3d, $n=4$ ), $\mathrm{IgG}^{+} / \mathrm{C} \mathrm{d}^{+}$(autoantibodies positive with both anti-IgG and anti-C3d, $n=73$ ), and RBC autoantibodies with alloantibodies $(n=9)$. For all subgroups, the frequencies of HLA-DRB1 and HLA-DQB1 alleles were calculated and compared to those from the control group. The odds ratios (ORs) and 95\% confidence intervals (CIs) were calculated to quantify the risk or 
protection conferred by the alleles in question, with a case: control ratio of 1:4. Our statistical procedures are similar to those of previous related studies ${ }^{[5,6]}$.

\section{Statistical analysis}

Chi-squared and Fisher exact tests were used to statistically compare the data between the case and control groups. The Bonferroni formula [adjusted $P$ value $\left.=1-(1-\text { crude } P)^{n}\right]$ was used to correct for multiple testing, and an adjusted two-sided $P$-value $\left(P^{\mathrm{a}}\right)<$ 0.05 was considered statistically significant. All statistical analyses were conducted using SPSS version 19.0 (SPSS Inc., Chicago, IL, USA).

\section{RESULTS}

To investigate the association of $\mathrm{RBC}$ autoantibodies with the presence of HLA-DRBl and HLADQB1 alleles, the 117 patients enrolled in the study

Table 1 Baseline of patients with RBC autoantibodies

\begin{tabular}{lccc}
\hline \multicolumn{1}{c}{ Subgroups } & $n(\%)$ & Age/yr, mean \pm SD & Gender-male $/ n(\%)$ \\
\hline $\mathrm{IgG}^{-} / \mathrm{C} 3 \mathrm{~d}^{+}$ & $4(3.4)$ & $59.1 \pm 2.3$ & $2(50.0)$ \\
$\mathrm{IgG}^{+} / \mathrm{C} 3 \mathrm{~d}^{-}$ & $34(29.1)$ & $58.2 \pm 25.5$ & $18(52.9)$ \\
$\mathrm{IgG}^{+} / \mathrm{C} 3 \mathrm{~d}^{+}$ & $70(59.8)$ & $52.6 \pm 21.7$ & $22(31.4)$ \\
autoantibodies with & $9(7.7)$ & $56.7 \pm 24.6$ & $1(11.1)$ \\
allo-antibody & $117(100.0)$ & $54.8 \pm 22.5$ & $43(36.8)$ \\
\hline
\end{tabular}

were divided into five subgroups. The mean age and male percentage in the case group was 54.8 years and $36.8 \%$, respectively; the other demographic details of the subgroups are shown in Table 1.

Patients in the $\mathrm{IgG}^{+} / \mathrm{C} 3 \mathrm{~d}^{+}$and $\mathrm{IgGG}^{-} / \mathrm{C} 3 \mathrm{~d}^{+}$subgroups showed higher frequency of the HLA-DRBI*14 allele, and no HLA-DRB $1 * 13$ allele was detected in the $\mathrm{C}_{3 \mathrm{~d}^{+}}$subgroup. After Bonferroni adjustment, the HLA-DRB ${ }^{*} 14\left(P^{\mathrm{a}}=0.0383\right)$ and HLA-DRB ${ }^{*} 13$ $\left(P^{\mathrm{a}}=0.0383\right)$ frequencies were significantly different from those of the control group only in the $\mathrm{C} 3 \mathrm{~d}^{+}$ subgroups. Compared to the frequency in the healthy control population, the total 117 patients, as well as the $\mathrm{IgG}^{+}$and $\mathrm{IgG}^{+} / \mathrm{C} 3 \mathrm{~d}^{+}$subgroups were found to be approximately two times more likely to carry the HLA-DRB ${ }^{*} 07$ allele, although these results did not reach statistical significance after $P$-value adjustment. Patients with both RBC autoantibodies and alloantibodies showed a higher frequency of HLA-DQB1*02, although this difference also did not reach statistical significance owing to the low sample size (Table 2).

\section{DISCUSSION}

A previous study conducted in the Netherlands revealed possible associations between anti-E production and HLA-DRBI*09 and between anti-S production and HLA-DRBI*07, and indicated that HLA-DRB1*15 may enhance the formation of multiple RBC antibody specificities ${ }^{[5]}$. Maluskova A et $a l .{ }^{[6]}$ subsequently confirmed the association of HLADRBI*15 with RBC antibody multi-responder status in the Czech population. In China, Dazhou W et al. ${ }^{[8]}$ observed that HLA-DRB1*07:01 was associated with the production of alloantibodies against RhD. However, all of these previous studies focused on the production of alloantibodies against specific antigens on the membrane of RBC, and there have been relatively few studies on the relationships of HLA status with the formation of RBC autoantibodies. The generation of RBC autoantibodies represents another important component of unexpected antibody generation that can cause autoimmune hemolytic disease contributing to substantial difficulties and poor outcomes for transfusion practice $^{[3]}$.

Our results demonstrated that among Chinese patients with confirmed presence of RBC autoantibodies, those with a $\mathrm{C} 3 \mathrm{~d}^{+}$result in the DAT showed a higher frequency of HLA-DRB1*14 compared to the healthy control population; moreover, none of these patients harbored the HLA-DRB1*13 allele. HLADRB $1 * 13$ has been proposed as a protective factor in patients with autoimmune disease, and patients with rheumatoid arthritis carrying the HLA-DRBI*13 allele were found to produce autoantibodies against anticitrullinated protein ${ }^{[9]}$. Although only a few RBC autoantibodies are known to induce autoimmune hemolytic disease, the presence of these autoantibodies inevitably causes huge transfusion problems owing

Table 2 HLA-DRB1 and HLA-DQB1 associations for patients with RBC auto-antibodies

\begin{tabular}{|c|c|c|c|c|c|c|c|}
\hline DAT results & Alleles & Case/n $(\%)$ & Control $/ \%$ & OR & $95 \% \mathrm{CI}$ & Crude $P$ & Adjust $P$ \\
\hline $\mathrm{DAT}^{+}(n=117)$ & $\mathrm{DRB} 1 * 07$ & $32(13.7)$ & 8.9 & 1.62 & $1.05-2.51$ & 0.027 & 0.2994 \\
\hline $\mathrm{IgG}^{+}$with or without $\mathrm{C} 3 \mathrm{~d}^{+}(n=113)$ & $\mathrm{DRB} 1 * 07$ & $32(14.2)$ & 8.9 & 1.70 & $1.10-2.64$ & 0.017 & 0.1998 \\
\hline \multirow[t]{3}{*}{$\mathrm{C} \mathrm{d}^{+}$with or without $\operatorname{IgG}^{+}(n=77)$} & $\mathrm{DRB} 1 * 04$ & $7(4.7)$ & 11.3 & 0.37 & $0.17-0.83$ & 0.012 & 0.1452 \\
\hline & $\mathrm{DRB} 1 * 13$ & $0(0.0)$ & 5.4 & - & - & 0.003 & 0.0383 \\
\hline & $\mathrm{DRB} 1 * 14$ & $22(14.7)$ & 7.0 & 2.22 & $1.29-3.84$ & 0.003 & 0.0383 \\
\hline $\mathrm{IgG}^{-} / \mathrm{C} 3 \mathrm{~d}^{+}(n=4)$ & $\mathrm{DRB} 1 * 10$ & $2(25.0)$ & 1.6 & 2.00 & $0.75-5.32$ & 0.032 & 0.3874 \\
\hline $\mathrm{IgG}^{+} / \mathrm{C} 3 \mathrm{~d}^{-}(n=40)$ & $\mathrm{DRB} 1 * 14$ & $11(14.5)$ & 7.0 & 2.16 & $1.00-4.66$ & 0.046 & 0.4578 \\
\hline $\mathrm{IgG}^{+} / \mathrm{C} 3 \mathrm{~d}^{+}(n=73)$ & $\mathrm{DRB} 1 * 07$ & $22(14.7)$ & 8.9 & 1.82 & $1.06-3.10$ & 0.027 & 0.2994 \\
\hline Autoantibodies with alloantibody $(n=9)$ & DQB1*02 & $7(38.9)$ & 12.5 & 4.46 & $1.37-14.46$ & 0.009 & 0.1109 \\
\hline
\end{tabular}


to cross-match incompatibility, and the alloantibodies cannot be readily distinguished from RBC autoantibodies, which show positive reactions with all screen and panel cells. Therefore, our results might offer a new direction towards a prophylaxis strategy for transfusion, especially for patients requiring several transfusions such as those with leukemia or hematological disease.

Our results may also provide insight into other well-known HLA-associated genetic predispositions such as RBC alloantibody or vaccinated immunization ${ }^{[10]}$; however, the low sample sizes and certain key factors such as environmental risk factors or smoking must be taken into account in interpreting these associations. Further large-scale studies are needed to comprehensively analyze the association between RBC autoantibodies and HLA alleles, even in the context of clinical significant autoimmune hemolytic disease, and detailed evaluation of the status of HLA class II alleles should be conducted to clarify whether HLA-DRB 1 alleles are protective or risk factors in terms of a predicted transfusion outcome.

In summary, this is the first study to demonstrate that HLA-DRB1 may play a key role in the formation of $\mathrm{C} 3 \mathrm{~d}$ positive RBC autoantibodies. In particular, our results suggest that HLA-DRB1*13 may serve as a protective factor and that HLA-DRB $1 * 14$ may be a risk factor for the production of anti-C $3 \mathrm{~d}^{+}$positive autoantibodies.

\section{References}

[1] Quist E, Koepsell S. Autoimmune hemolytic anemia and red blood cell autoantibodies. Arch Pathol Lab Med,
2015;139(11):1455-8.

[2] Barcellini W. The clinical dilemma and management of red cell autoantibodies. Expert Rev Hematol, 2016;9(4):325-7.

[3] Lin YS, Chang JS, Qiu Y, et al. Incidence of unexpected red blood cell antibodies in the north of China. Asia-Pacific Journal of Blood Types and Genes, 2017;1(1):17-24.

[4] Petz LD, Garratty G. Immune hemolytic anemias. 2nd edition. London: Churchill Livingstone, 2004: 167-76.

[5] Schonewille H, Doxiadis, II, Levering WH, et al. HLADRB1 associations in individuals with single and multiple clinically relevant red blood cell antibodies. Transfusion, 2014;54:1971-80.

[6] Maluskova A, Mrazek F, Pauliskova M, et al. Association of HLA-DRB1 and HLA-DQB1 with red-bloodcell alloimmunization in the Czech population. Vox Sang, 2017;112(2):156-62.

[7] Zhou XY, Zhu FM, Li JP, et al. High-resolution analyses of human leukocyte antigens allele and haplotype frequencies based on 169,995 volunteers from the China Bone Marrow Donor Registry Program. PLoS One, 2015;10(9):e0139485.

[8] Dazhou W, Manni W, Dan Z, et al. Analysis of the relationship between the HLA-DRB1 alleles and the production of RhD antibody. Chin J Blood Transfusion, 2014;27(12):1290-2.

[9] Bettencourt A, Carvalho C, Leal B, et al. The protective role of HLA-DRB1*13 in autoimmune diseases. J Immunol Res, 2015;2015:948723.

[10] Posteraro B, Pastorino R, Di Giannantonio P, et al. The link between genetic variation and variability in vaccine responses: systematic review and meta-analyses. Vaccine, 2014;32:1661-9.

(Received 18 April 2017, Revised 28 April 2017, Accepted 10 May 2017) 